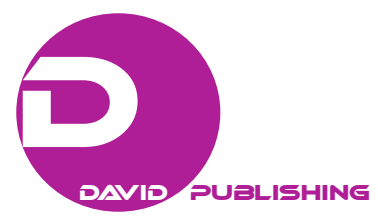

\title{
The Path Choice and Method of Chinese Rural Governance Ethics Research
}

\author{
Ang Liu \\ Nanjing Normal University Rural Culture Promotion Research Center, Nanjing, China \\ Nanjing Normal University, Nanjing, China
}

\begin{abstract}
The 19th National Congress of the Communist Party of China put forward the strategy of Rural Revitalization. Effective governance is the key to rural revitalization. Effective rural governance needs to properly deal with the ethical relationship in the process of governance. The study of rural governance ethics in China includes three kinds of path choices. They are paradigm shift from top-down to bottom-up, organic integration of special and general, and interweaving between tradition and modernity. The paradigm shift from top-down to bottom-up needs to be done by methods, such as the use of the relationship between theory and practice, the change from abstract research to applied research, and the implementation of fieldwork surveys. Special and general organic integration depends on methods, such as the law of contradiction specificity and universality, the cooperation of induction and deduction, the operation of case studies, etc. The interweaving of tradition and modernity requires methods to be realized, such as the negation of negation, moral narrative, and non-intrusive research.
\end{abstract}

Keywords: rural governance ethics, path choice, method

\section{Introduction}

The path of China's rural governance ethics research mainly includes the following three types. Firstly, the paradigm shift from top-down to bottom-up which is accomplished through the use of the relationship between theory and practice, changes from abstract research to applied research, and the implementation of fieldwork. Secondly, special and general organic integration which relies on the laws of contradiction and universality, the cooperation of inductive and deductive, and the operation of case studies. Thirdly, the interweaving of tradition and modernity which is achieved by means of negative negation, moral narrative, and non-intrusive research.

\section{Paradigm Shift From Top-Down to Bottom-Up}

The paradigm shift from top-down to bottom-up is the primary path for the study of rural governance ethics in China. The realization of this path needs to be done by means of methods, such as the application of the relationship between theory and practice, the change from abstract research to applied research, and the implementation of field investigations.

\footnotetext{
Ang Liu, Ph.D., lecturer, School of Marxism Studies, Nanjing Normal University; Nanjing Normal University Rural Culture Promotion Research Center, Nanjing, China.
} 


\section{The Relationship Between Theory and Practice}

The practical viewpoint as the primary and basic viewpoint of Marxist philosophy constitutes the basis for the transformation of the paradigm of rural governance ethics in China today. The change from the top-down to the bottom-up research method is the view of scientific practice. The change of research method from "top-down" to "bottom-up" is a reasonable grasp of scientific practice view.

"The relationship between theory and practice constitutes the fundamental problem of Marxist philosophy" (Zhang, 2015, p. 18). In Marx's view, practice is the foundation of theory. The construction of all theoretical knowledge is inseparable from the exploration of practice. He emphasizes that "all social life is essentially practiced. Any mystery that leads theory to mysticism can be reasonably resolved in human practice and in understanding this practice" (Marx \& Engels, 2009, p. 501). At the same time, Marx also pointed out,

Do people's thinking have objective truth. This is not a theoretical problem, but a practical problem. People should prove the truth of their thinking in practice, that is, the reality and strength of one's own thinking, the shore of one's own thinking. (Marx \& Engels, 2009, p. 500)

He believes that people know the world correctly. Its key is whether this understanding can be tested by practice. In turn, all the theories created by human beings must be tested by practice to become truth. The research without practice is not scientific. Of course, while emphasizing the first nature of practice, Marx did not ignore the promotion of theory to practice. He stressed that a theory once which has mastered the masses will also become a material force. As long as the theory convinces people, the theory can grasp the masses. If the theory is thorough, it can convince people. The so-called thoroughness is to grasp the root of things. The root of man is the person himself (Marx \& Engels, 2009, p. 11). Marx clearly recognizes the dialectical relationship between theory and practice. It gives methodological guidance for the study of rural governance ethics. The dialectical relationship between theory and practice in Marxist philosophy requires us that the study of rural governance ethics must be transformed from a top-down research approach that focuses on theoretical analysis to a bottom-up study based on concrete practice way. Through rural specific practice, he seeks ethical governance measures. Then, he tests through practice whether the rural governance ethics theory can truly satisfy the villagers' desires and represent the legitimate interests of the villagers. At the same time, relevant theories have reference to rural practice. It can enrich and improve the ethics theory of rural governance through the theoretical research of predecessors.

\section{From Abstract Research to Applied Research}

The transformation of the rural governance ethics research paradigm from top-down to bottom-up is accompanied by changes in the academic community from abstract research to application-oriented research.

Abstract research and applied research are the research fields that often appear in sociology of philosophy. Some researchers fascinated the essence of human social life and were driven to explain it. They like to discover the meaning through the appearance of sly. Pure research in all fields of science is sometimes justified by seeking "pure knowledge" (Al Barbie, 2009, p. 26). At the same time, another group of researchers may be inspired by their research goals. They want to show what they have learned. And they want to put their knowledge about society into action (Al Barbie, 2009, p. 26). The two studies should be in a reasonable equilibrium and serve the community together. But for a long time, influenced by the top-down research method, most researchers disdain or even smash application research. They believe that the so-called applied research cannot be called true science, which leads to serious obstacles to the development of bottom-up applied research. 
The results of China's rural governance ethics research in the last century are rare, because the purely academic inquiry represented by meta-ethics or critical analytic ethics has always been one of the mainstreams of Western ethics throughout the 20th century. In China, the voice of "ethics should return to life" and the study of moral life history and daily ethical life, among the research results of a large number of normative ethics and analytical ethics, can only be regarded as a small episode (Wang, 2007a). In the 21st century, more and more researchers are beginning to discover that past abstract research seems to be in a dead end. They cannot be closely integrated with the real society, let alone solving social realities. Under the influence of this background, the research paradigm of philosophy and social sciences began to pick out from the top-down study. It gradually tries to bottom-up research methods. Then, it rise of applied research. The study of rural governance ethics is gradually carried out under the guidance of this research method.

\section{Field Investigation}

The field survey is the basic method of the bottom-up research paradigm, which can collect first-hand information very well. It can accurately grasp the problems encountered in rural governance and fully understand the customs of rural society. This has a non-negligible role in the construction of rural governance ethics.

Fieldwork, also known as field research, is a life-long context in which to study phenomena. It collects data in the form of participating observations and unstructured interviews, and through the qualitative analysis of these materials, understands and explains the sociological research methods of phenomena (Feng, 2001, p. 238). This research method first appeared in anthropological research. It was gradually accepted by sociology and other disciplines, and gradually became one of the basic methods of philosophical humanities and social science research. It broke the top-down research method of heavy theoretical analysis and light empirical research that has been formed for a long time. It is a typical bottom-up research paradigm. In general, the field survey requires three stages, "the development of the survey plan and the design of the questionnaire; the field implementation of the field survey; the collation of interview recordings and the data processing and statistical analysis of the questionnaire" (Wang, 2008, pp. 24-30). Each stage is interlocked to form a complete field research data.

The study of rural governance ethics must involve the specific reality of the countryside. The field investigation can meet this demand and help to collect the first-hand information of rural governance. This lays the foundation for theoretical analysis. At the same time, with the expansion of the research area and the dispersion of the research group, the survey often encounters "entry barriers", such as language barriers, local protection, refusal to cooperate, etc. (Zheng, 2003). Faced with these challenges, researchers need to learn to adapt and try to enter the country from multiple angles. This kind of trial process itself is also a field survey of rural governance ethics.

\section{The Organic Integration of Special and General}

In the context of China's vast geography, we must follow the special and general organic integration path. The achievement of this research path depends on the laws of contradiction and universality, the cooperation of inductive and deductive, and the operation of case studies.

\section{The Particularity and Universality of Contradiction}

Contradiction is the inevitable state of the development of things, everywhere and everywhere. Its 
particularity and universality also accompany it. The speciality in the study of rural governance ethics is the particularity of contradiction. However, in general, it belongs to the universality of contradictions. The two complement each other and eventually merge to make the contradictions resolved.

The particularity and universality of contradiction is one of the fundamental methods of cognition. Specifically, the particularity of contradiction means the nature and status of contradiction and the specific forms of resolving contradictions have their own characteristics (X. L. Li, Wang, \& H. C. Li, 2004, p. 183). The universality of contradiction is mainly manifested in two aspects. Firstly, contradictions exist in the development of everything. Secondly, there is a contradictory movement from beginning to end in the development of each thing (Mao, 2009, p. 305). The particularity of contradiction contains the universality of contradiction. The universality of contradiction runs through the particularity and is manifested through speciality. At the same time, commonity is the particularity of contradiction as the individuality contains the universality of contradiction. The commonality lies in the individuality. The universality of contradiction runs through the particularity of contradiction. The commonality takes the individuality. The commonality and individuality are connected with each other. Reciprocally, they transform each other under certain conditions (X. L. Li et al., 2004, p. 185).

Our country is vast. Every village has its own customs. There must be differences between different villages. Any kind of rural governance ethics model is constructed on the basis of various regional specialities that cannot be cloned (Wang, 2007a). In this sense, there is an unavoidable contradiction between the speciality of rural governance ethics and the generality that can be universally applied. However, the particularity of the contradiction and the law of universality tell us that the commonality is in the individuality and the personality is taken, between the special and general values of villages that seem to be incompatible. In fact, there is an opportunity for organic integration between them. Any special rural governance path that meets ethical requirements involves the recognition of the respect and value of the rural subject. Although the rural governance models in different regions are different, the purpose of governing the villages is the same. Therefore, in the process of studying rural governance ethics, we must explore the general values behind the villages and strive to achieve organic integration.

\section{Induction and Deduction}

No matter it is from the special sum of the village to the general value of the summary or from the general value to the special deductive reasoning of the village, both of them are two specific methods of organically blending the special and general values of the village. They play a pivotal role in the rural governance ethics.

Induction is the discovery of a model that starts from the individual to achieve generality and from a series of specific observations (Al Barbie, 2009, p. 23). At the same time, deduction is a way of thinking, whether it is from the general to the individual, from the logical or theoretically expected model to the observation of the expected model (Al Barbie, 2009, p. 24). Inductive and deductive are just the opposite. Inductive sums up the general law from special. The deduction does push the special existence through general rules. The two are right and wrong and complement each other. They are all scientific methods of understanding things, a combination of the two. This will help researchers to establish a complete and true understanding of the research object. At the same time, in terms of the human cognitive process, it is from individual to general and common. Then, it uses this general and common thing to study new, individual, special things. It complements 
and enriches the realization of this common understanding of the essence. Scientific understanding is the process of this cycle, deepening and deepening (X. L. Li et al., 2004, p. 185). Inductive and deductive is the driving force of this process.

The organic integration of village speciality and general value in rural governance ethics depends on the application of induction and deduction. We should fully tap the commonalities between different villages and find the difficulties and solutions that we have encountered in our governance. Then we reason out the ethical principles of rural governance with general value. At the same time, we also need to work hard to translate governance norms of general value into villages of different types. As far as possible, it provides practical guidance for rural governance in different regions, constantly sums up the general value through the village, and then deducts the special village from the general value, and recurs and deepens. In the end they achieve an organic integration of the two. Thus we enrich the study of rural governance ethics.

\section{Case Study}

In the face of thousands of different types of villages in China, it is difficult for researchers to find out the general value that can be adapted to all villages. However, according to the case study, it is reasonable to find the village speciality of some villages, and then find the general value of the national rural governance ethics through the method of induction.

As a basic research method of sociological experience research, it is a long history for the case studies. In the past 100 years, through the joint promotion of anthropology and sociology, case studies have become one of the most important research orientations in humanities and social science research ( $\mathrm{Lu} \& \mathrm{Li}, 2007)$. In recent years, this method has been favored by applied ethics researchers and has become one of the classic research methods of philosophy and social sciences. For the case in the case study, it also means the system with boundary (Stake, 2005, p. 444). The boundary refers to the difference between a case and another case and its environment ( $\mathrm{Lu} \mathrm{\&} \mathrm{Li}, 2007)$. The system emphasizes that the components of a case constitute a relatively self-contained unit ( $\mathrm{Lu} \& \mathrm{Li}, 2007)$. Research on this bounded system has resulted in case studies.

Although the conclusions of the case study have obvious village specialities, it is not possible to directly become a general value. However, if we describe and analyze a typical village by concrete description, reveal its particularity as a case, and provide valuable information for a comprehensive analysis of the general laws of rural development in China, whether from the feasibility of research methods or the results of research, in terms of reliability, it is worthy of recognition (Wang, 2007b). In the process of Chinese rural governance ethics research, the special value of case study should be correctly viewed. Through the methods of induction and deduction, the relationship between the general value and the general value should be handled to achieve organic integration.

\section{Intertwined Between Tradition and Modernity}

As an ancient country with a long history of civilization, the realization of the integration of tradition and modernity is the path that must be realized in the study of rural governance ethics. The resolution of this path requires negation of negation laws, moral narratology, and non-intrusive research.

\section{Negative Negation}

The negative law of negation is the inevitable process of the development of things. It is a spiral rise. The traditional experience in the study of rural governance ethics must be a valuable resource for rural 
governance. It has played an irreplaceable role in a certain period of time. After the process of "affirmation-negation-negation of negation", it will continue to provide value support for rural governance.

The negative law of negation shows that intrinsic contradiction or inner negative power promotes the transformation of existing things into their own opposites. It is sure to reach a denial of itself, and then a new affirmation by negation, that is, a negation of negation (X. L. Li et al., 2004, p. 187). Affirmation and negation are two sides of things. They are mutually exclusive and mutually infiltrated. They constitute a dialectical relationship of unity of opposites. Therefore, the negation of negation is a dialectical negation. It is self-denial through the inner contradictory movement of things, that is, denying oneself and self-rejecting to achieve self-movement and self-development. It is the opportunity and driving force for the development of things, and it is the essence of the revolution of dialectical criticism (X. L. Li et al., 2004, p. 188). From this perspective, this dialectical negative view becomes a methodological principle for observing and analyzing all problems (X. L. Li et al., 2004, p. 188). It requires us to look at things from a developmental perspective. In the process of affirmative understanding of things, we need to consider the power of negation at the same time. And we must not only make a one-sided positive understanding.

With the continuous deepening of the modernization process, the rural society in China is no longer described in the Tang poetry and Song poetry. The closedness and stability of traditional local ethics are broken. The marketization process has injected fresh, contemporary, and ethical values into the blood of the new era (Wang, 2010). This provides a contemporary atmosphere for the current research on rural governance ethics. However, we cannot ignore the traditional experience of traditional rural governance ethics. The "Time Breath" is able to provide ethical support for today's rural governance. To a certain extent, this is inseparable from the negation of the negative experience of traditional rural governance. Therefore, it attempts to completely break with the local ethical tradition. This will only make the new local ethics lose its foundation and become a conceptual framework of empty objects or suspend useless moral preaching (Wang, 2010). Ultimately, it is not conducive to the development of rural governance ethics research. All in all, it is an inevitable choice for the current rural governance ethics research to successfully graft the traditional rural governance experience and modern atmosphere.

\section{Moral Narratology}

Moral narratology is a bridge to graft the traditional experience of rural governance ethics and the "temporal atmosphere". This research method can find a reasonable point of convergence between rural tradition and reality, thus optimizing the status quo of rural governance.

Moral narratology is based on a specific moral context. It is a kind of moral narrative created by combing the moral life experience of a particular moral community or ethical community and the generation and evolution of its moral concepts (Wang, 2007a). This research method has been inspired by McIntyre's related research. As early as in the 1980s, McIntyre asserted that the moral rationality arguments demonstrated since the Enlightenment in the 18th century must fail. He believed that this movement has led to the interruption of tradition, when people do not have the "bundling" of traditional experience. They have only a fragment of a conceptual framework, and many have lacked the context in which they derive their meaning (McIntyre, 2003, p. 2). Therefore, it is impossible to find the "temporal atmosphere". Thus the moral rationality argument in this context will inevitably fail.

It is worth noting that "the method of moral narratology is not just a historical morality. Its more critical feature is its contextualist appeal to the unique diversity of human moral life experience and knowledge, 
traditional pedigree, and moral knowledge understanding (Wang, 2007a). This can fully reflect the traditional experience of the research object. Of course, this does not mean a compromise to any moral relativism. On the contrary, it emphasizes the relative diversity of human moral life experience itself and the rich diversity of human moral culture traditions (Wang, 2007a). Furthermore, it makes a more reasonable and accurate grasp of the "temporal atmosphere" of the research object. From the point of view, the successful grafting of tradition and modernity in the study of rural governance ethics cannot leave the research method of moral narratology. Therefore, in the study of rural governance ethics in China, the use of moral narratology to graft the tradition and modernity of the research object can not only avoid the phenomenon of the disconnection between reality and history, but also find the historical development context for the research.

\section{Non-invasive Study}

In general, the tradition of rural governance ethics is far from the modern one. The respondents may not have a more accurate understanding of this. Even if the respondent is fortunate enough to have a clear memory of this, it is also possible to choose not to tell the researcher the true situation and answer for various considerations. This practice often puts the researcher in a passive state, so that it is impossible to find true traditional experience and "temporal atmosphere". In turn, the two cannot be successfully grafted, which ultimately affects the research results.

Faced with the above situation, non-intrusive research methods often have breakthroughs. The so-called non-intrusive research method is a method of studying social behavior without affecting the research object (Al Barbie, 2009, p. 24). The advantage of this approach is that it reduces the likelihood that the respondent will deliberately create false information for some reason. It does not require the respondent to directly answer the answer. Instead, it relies on the investigator's observation and analysis capabilities to arrive at more realistic findings. For example, investigators want to know which seat in a fixed classroom is more popular with students. The usual survey is to find a group of students to conduct a questionnaire or interview. However, students may choose the first row for reasons such as good students prefer to sit in the first row. This will lose the authenticity of the investigation. Non-intrusive research does not require direct inquiry to students. Instead, you can get a more realistic answer by observing which row of tables and chairs are worn out in the classroom.

In the traditional and modern surveys of rural governance ethics, whether the respondents are intentional or indeed vague, if they are wrong, they will affect their successful grafting. Therefore, the introduction of non-invasive research can effectively improve the authenticity of the survey results. This collects more realistic information for rural governance ethics research. Of course, emphasizing the role of non-invasive research is not a denial of interventional research. Interventional research in many places still has its irreplaceable advantages. Only by effectively combining the two will the authenticity of the research results be maximized.

\section{Conclusions}

The study of rural governance ethics in China includes three kinds of path choices. They are paradigm shift from top-down to bottom-up, organic integration of special and general, and interweaving between tradition and modernity. The comprehensive use of these paths can effectively improve the ethical research of rural governance in China. It can promote the level of rural governance in China. At the same time, it can promote the modernization of national governance system and governance capacity. 


\section{Acknowledgements}

Funding for this work was provided through the Project of Philosophy and Social Science Research in Colleges and Universities in Jiangsu Province (Project No. 2019SJB072), the National Philosophy and Social Science Foundation (Project No. 15ZDB014), the Jiangsu Province Social Science Foundation Youth Program

(Project No. 18ZXC003), key research base of philosophy and social science in universities in Jiangsu Province: Rural Culture Revitalization Research Center (Project No. 2018ZDJD-A005).

\section{References}

Al Barbie. (2009). Social research methods (11th ed.). (Z. Q. Qiu, Trans.). Beijing, China: Huaxia Publishing House. Feng, X. T. (2001). Sociology research methods. Beijing, China: China Renmin University Press.

Li, X. L., Wang, Y., \& Li, H. C. (2004). Principles of dialectical materialism and historical materialism (5th ed.). Beijing, China: China Renmin University Press.

Lu, H. L., \& Li, X. (2007). How to get out of the case: From case study to extended case study. China Social Sciences, (1), 118-130, 207-208.

Marx, K., \& Engels, F. (2009). Marx and Engels anthology, Vol. 1. Beijing: People's Publishing House.

Mao, Z. D. (2009). Mao's anthology, Volume 1. Beijing, China: People's Publishing House.

McIntyre. (2003). Following virtue (J. J. Song, Trans.). Nanjing: Yilin Press.

Stake, R. E. (2005). Qualitative case studies. In N. K. Denzin and Y. S. Lincoln (Eds.), The sage handbook of qualitative research (pp. 443-454). Thousand Oaks, California: Sage Publications.

Wang, L. L. (2007a). Research on Chinese local ethics and its methods in the period of social transformation. Philosophical Research, (12), 79-84.

Wang, L. L. (2007b). The resource significance of some classical theories on rural economic ethics research. Ethics Research, (1), 74-78.

Wang, L. L. (2008). Local ethics: An exploration of "local moral knowledge” in an interdisciplinary perspective. Beijing: People's Publishing House.

Wang, L. Z. (2010). From local ethics to new rural ethics: The traditional features and modern transformation of Chinese rural ethics. Toyo Ethics and Culture-18th Hanzhong Proceedings of the International Conference on Ethics. Chinese Ethics Society, Korean Ethics Society, Korea Education Evaluation Institute.

Zhang, X. H. (2015). Modern practical philosophy and historical materialism. Research of Philosophy, (3), 18-23.

Zheng, X. (2003). Field investigation and site entry: Discussion on empirical methods of contemporary Chinese studies. Journal of Nanjing University (Philosophy, Humanities \& Social Sciences), (3), 52-61. 